\title{
As patentes nas universidades: análise dos depósitos das universidades públicas paulistas
}

José Roberto Plácido Amadei

Mestre em engenharia de produção pela Universidade Federal de São Carlos, bibliotecárioda Faculdade de Odontologia de Bauru-Universidade de São Paulo, Bauru/SP.

E-mail: $\underline{\text { amadei@usp.br }}$

Ana Lúcia Vitale Torkomian

Doutora em administração de empresas pela FEA/USP. Professora do Departamento de Engenharia de Produção da UFSCar, São Carlos/SP

E-mail: torkomia@ufscar.br

\section{Resumo}

Este artigo analisou os depósitos das universidades públicas paulistas no Instituto Nacional de Propriedade Industrial (Inpi) no período de 1995-2006, perfazendo 672 registros. Foram consideradas as seguintes: Universidade Estadual de Campinas, Universidade de São Paulo, Universidade Estadual Paulista, Universidade Federal de São Carlos e Universidade Federal de São Paulo. Com os resultados obtidos verificou-se um avanço, mesmo que instável, do número de depósitos de patentes advindas das universidades e o adiantamento da Unicamp em relação às demais na quantidade de patentes depositadas, responsável por $60 \%$ do total de registros. Como conclusão vale destacar que o fortalecimento das políticas internas das universidades relacionadas à propriedade industrial acarretará maior índice de proteção das invenções acadêmicas, garantindo os direitos sobre a invenção, incentivando a realização de novas pesquisas e, através de mecanismos efetivos, viabilizando a transferência da tecnologia produzida nas universidades para o setor produtivo.

\section{Palavras-chave}

Universidades. Patentes Acadêmicas. Propriedade intelectual.

\section{University patents: analysis of the deposits of paulista public universities (1995-2006)}

\begin{abstract}
The present work analyzed the deposits of Paulista public universities with the National Industrial Property Institute (Inpi), in the period 1995-2006, totaling 672 records. The universities taken into account, in this study, were: Campinas State University, University of São Paulo, Paulista State University, São Carlos Federal University and São Paulo Federal University. Based on the results achieved, an improvement was verified, although unstable, in the number of patent deposits from universities. As for the quantity of patents deposited, Unicamp was ahead of other universities, being responsible for $60 \%$ of total records. Another characteristic found, was the diversity in which deposits are made. As a conclusion, it should be highlighted that the strengthening of internal policies of universities related to industrial property, will cause a higher level of protection for academic inventions, guaranteeing patent rights, stimulating new research and through effective mechanisms, transfer the technology produced in universities to the productive sector.
\end{abstract}

\section{Keywords}

Universities. Patents. Intelectual property. 


\section{INTRODUÇÃO}

A intensificação da concorrência entre as empresas que atuam no mercado nacional e internacional é consequência da liberalização comercial e econômica, a chamada globalização. Tal concorrência tem se transformado em um ambiente análogo ao da seleção natural das espécies, em que somente os mais aptos sobreviverão. Em consequência, as organizações se voltam para um monitoramento constante de suas atividades e da viabilidade dos produtos e serviços oferecidos por elas no mercado.

Nesse contexto, a inovação tecnológica nas empresas tem se configurado como fator fundamental para a manutenção de suas atividades e para o crescimento e desenvolvimento do país. Inovação, segundo o manual de Oslo (OECD, c1997), consiste na implementação de um bem ou serviço novo ou significativamente melhorado, ou um processo, ou um método de marketing, ou um novo método organizacional.

Segundo Hsu (2005), “o processo de inovação requer vários tipos de tecnologia e conhecimento oriundos de diferentes fontes, incluindo indústria, empresas, laboratórios, institutos de pesquisa e desenvolvimento, academia e consumidores".

Engajadas dentro das estratégias governamentais relacionadas à política científica e tecnológica do país, as universidades apresentaram nos últimos anos substancial amadurecimento no desempenho de suas atividades direcionadas ao desenvolvimento econômico. Tal amadurecimento é evidenciado pela criação de estruturas internas às universidades com objetivo de facilitar o transbordamento do conhecimento científico para o meio empresarial, mediante o desenvolvimento de pesquisas conjuntas entre universidades e empresas, a geração de spinoffs acadêmicos e o licenciamento de patentes depositadas pelas universidades (TORKOMIAN, 1997).

As patentes acadêmicas têm ganhado notoriedade no estabelecimento de políticas públicas em um ambiente caracterizado por inovações (HAASE; ARAÚJO; DIAS, 2005).

A patente é um título de propriedade industrial sobre invenção ou modelo de utilidade. Um prêmio outorgado pelo estado como recompensa ao inventor. $\mathrm{O}$ registro de patente garante ao inventor certa segurança nas negociações entre ele e a parte interessada em comprar determinada tecnologia para que possa ser aplicada em algum setor industrial.

No registro de patente o inventor é obrigado a revelar detalhadamente todo o conteúdo técnico do invento, sendo possível para qualquer técnico reproduzi-lo em laboratório. As patentes são depositadas nos institutos responsáveis pela proteção; estima-se que $70 \%$ da informação contida nos documentos de patentes não estão disponíveis em qualquer outra fonte de informação (WIPO, c2007). "Patente ou propriedade intelectual [...] protege a inteligência, a criatividade, o invento, a pesquisa e pressupõe, na sua adoção, uma interação com a comunidade internacional" (LOPES, 1999).

Sabino (2007) demonstrou em recente estudo que a proteção à propriedade intelectual, por meio das patentes, estimula o desenvolvimento econômico do país e inovações tecnológicas que gerem riqueza e bem-estar geral, desde que as leis de proteção patentária sejam bem aplicadas.

Este estudo apresenta uma caracterização das patentes depositadas pelas universidades públicas paulistas no Instituto Nacional de Propriedade Industrial (Inpi), órgão nacional responsável pelo registro da propriedade industrial. As características analisadas são as seguintes:

- a evolução do número de depósitos ao longo dos anos;

- a participação de cada universidade no número total de depósitos realizados;

Ci. Inf., Brasília, v. 38, n. 2, p. 9-18, maio/ago. 2009 
- a relação dos depósitos com o número de pesquisadores atuantes nas universidades e seus programas de pós-graduação;

- as áreas em que, segundo a classificação de domínios tecnológicos da OST $^{1}$, as universidades se destacam.

\section{AS UNIVERSIDADES COMO CENTROS DE INOVAÇÃO}

Por muitos séculos as universidades foram vistas como locais alheios aos acontecimentos sociais e econômicos. Todas as mudanças que ocorreram na função das universidades se deram lentamente. Tais mudanças, segundo Etzkowitz (2003), foram possibilitadas por uma primeira revolução acadêmica, ocorrida no final do século XIX, em que as universidades adicionaram às suas funções atividades de pesquisa. Uma segunda revolução acadêmica permitiu às universidades direcionar suas atividades a fim de contribuir para o desenvolvimento econômico. Esta teve início com a criação do Massachusetts Institute of Technology (MIT), em 1862. Desde então, esse modelo passou a ser transferido para outros centros universitários.
O quadro 1 apresenta as missões das universidades dentro de cada momento de sua história e nas revoluções acadêmicas apresentadas por Etzkowitz (2003).

As universidades marcaram a trajetória de inovação de muitos países chamados hoje de desenvolvidos. Cita-se como exemplo o apoio dado à pesquisa básica nas universidades norte-americanas após a Segunda Guerra Mundial. Uma das estratégias do governo norte-americano para recuperar sua economia e emergir como potência mundial foi o fornecimento de fundos para a pesquisa universitária e o estreitamento das relações das universidades com a indústria (MOWERY; ROSENBERG, 2005). Nos Estados Unidos, as universidades são consideradas elemento estratégico para o desenvolvimento econômico e tecnológico do país.

Em Taiwan, a estrutura industrial tem obtido benefícios da interação entre o governo e os institutos de pesquisas nacionais. Institutos de pesquisa, academia e indústria constituem os principais elementos em um sistema nacional de inovação (HSU, 2005).

\section{QUADRO 1}

\section{Expansão da missão das universidades}

\begin{tabular}{l|l|c}
\hline Ensino & Pesquisa & Universidade empreendedora \\
\hline $\begin{array}{l}\text { Preservação e disseminação do } \\
\text { conhecimento }\end{array}$ & Primeira revolução acadêmica & Segunda revolução acadêmica \\
\hline $\begin{array}{l}\text { Novas missões geram conflito } \\
\text { de interesses } \\
\text { antigas }\end{array}$ & Duas missões: ensino e pesquisa & $\begin{array}{c}\text { Três missões: desenvolvimento } \\
\text { econômico e social, missões } \\
\text { continuam }\end{array}$ \\
\hline
\end{tabular}

Fonte: Etzkowitz (2003).

\footnotetext{
${ }^{1}$ A OST, L’Observatoire des Sciences et des Techniques, é uma organização francesa que tem por missão elaborar indicadores de pesquisa e inovação tecnológica . A classificação de domínios tecnológicos foi criada a partir da Classificação Internacional de Patentes, agrupando os registros com classificações similares em um mesmo domínio ou subdomínio. A Fundação de Amparo à Pesquisa do Estado de São Paulo (Fapesp) utiliza tal classificação para elaboração de seus indicadores patentários.
}

De acordo com os resultados alcançados com a pesquisa "Mobilização Brasileira pela Inovação" - Mobit (ABDI, 2007), em que as políticas para inovação tecnológica de sete países foram estudadas (Estados Unidos, Canadá, Irlanda, Reino Unido, França, Finlândia e Japão), têm ocorrido muitas discussões sobre a adaptação das universidades 
ante as mudanças ocorridas no cenário econômico. Entre as pautas dos debates, a relação universidadeempresa tem ganhado destaque. A aproximação da academia com o meio produtivo tem se caracterizado como a estratégia fundamental para a inovação.

Como consequência desse novo papel atribuído às universidades, ocorreu um crescimento de ações governamentais que teve por finalidade estabelecer políticas e diretrizes que auxiliem a academia nesse processo. As questões sobre propriedade intelectual e transferência de tecnologias passaram a ocupar as pautas das discussões dos responsáveis pelas políticas das universidades, sobretudo nas universidades públicas.

No Brasil a gestão da propriedade intelectual dentro das universidades mereceu destaque nos últimos anos, sobretudo após a Lei de Inovação, aprovada em dezembro de 2004 e regulamentada pelo decreto 5.563, de outubro de 2005 (TORKOMIAN, 2009).

\section{METODOLOGIA}

Esta pesquisa consistiu no levantamento das informações patentárias na base nacional de patentes do Inpi. Foram analisados os registros de depósitos de patentes realizados pelas universidades públicas paulistas ao longo de 11 anos (1995-2006), deixando-se de incluir a Universidade Federal do $A B C$, em razão de sua recente criação. A escolha do estado justifica-se por São Paulo abrigar importantes universidades responsáveis, entre os anos de 2002 e 2004 , por 49,8\% dos depósitos realizados por todas as universidades do país (Inpi, 2007).

As universidades aqui analisadas já foram objeto de estudos anteriores que demonstraram sua relevância e o estágio de amadurecimento diferenciado que apresentam na que tange à gestão da propriedade intelectual (GARNICA,2007).

As estratégias de buscas utilizadas na base nacional de patentes e as universidades compreendidas neste estudo estão presentes no quadro 2. Para análise dos domínios e subdomínios tecnológicos, os números de classificações de cada registro foram substituídos pela classificação OST, anteriormente mencionada.

\section{RESULTADOS E DISCUSSÃO}

A figura 1, a seguir, revela o crescimento do número de patentes depositadas pelas universidades paulistas entre 1995 e 2006, período em que ocorreram 672 depósitos de pedidos de patentes. O gráfico demonstra a importância que as patentes vem ganhando no âmbito acadêmico nos últimos anos, tendo seu ápice no ano de 2005, provavelmente pelo estímulo da Lei de Inovação. O crescimento mais significativo ocorreu no ano de 2002, em que os depósitos saltaram de 36 em 2001 para 96 no ano seguinte, possivelmente em decorrência de

\section{QUADRO 2}

Estratégia de busca utilizada para recuperação dos registros de depósitos de patentes na base do Inpi

\begin{tabular}{l|l|r}
\hline Instituição & Estratégia para o campo Depositante & Período recuperado \\
\hline UFSCar & ufscar OR (universidade AND federal AND sao carlos) & $1995-2006$ \\
Unesp & unesp OR (universidade AND estadual AND paulista) & $1995-2006$ \\
Unicamp & unicamp OR (universidade AND campinas) & $1995-2006$ \\
Unifesp & unifesp OR (universidade AND federal AND sao paulo) & $1995-2006$ \\
USP & usp OR (universidade AND sao paulo) & $1995-2006$ \\
\hline
\end{tabular}

${ }^{2}$ Nesta expressão também foram recuperados os registros da Universidade Federal de São Paulo, os quais foram eliminados 
FIGURA 1

Depósito de patentes das universidades públicas paulistas no período 1995-2006

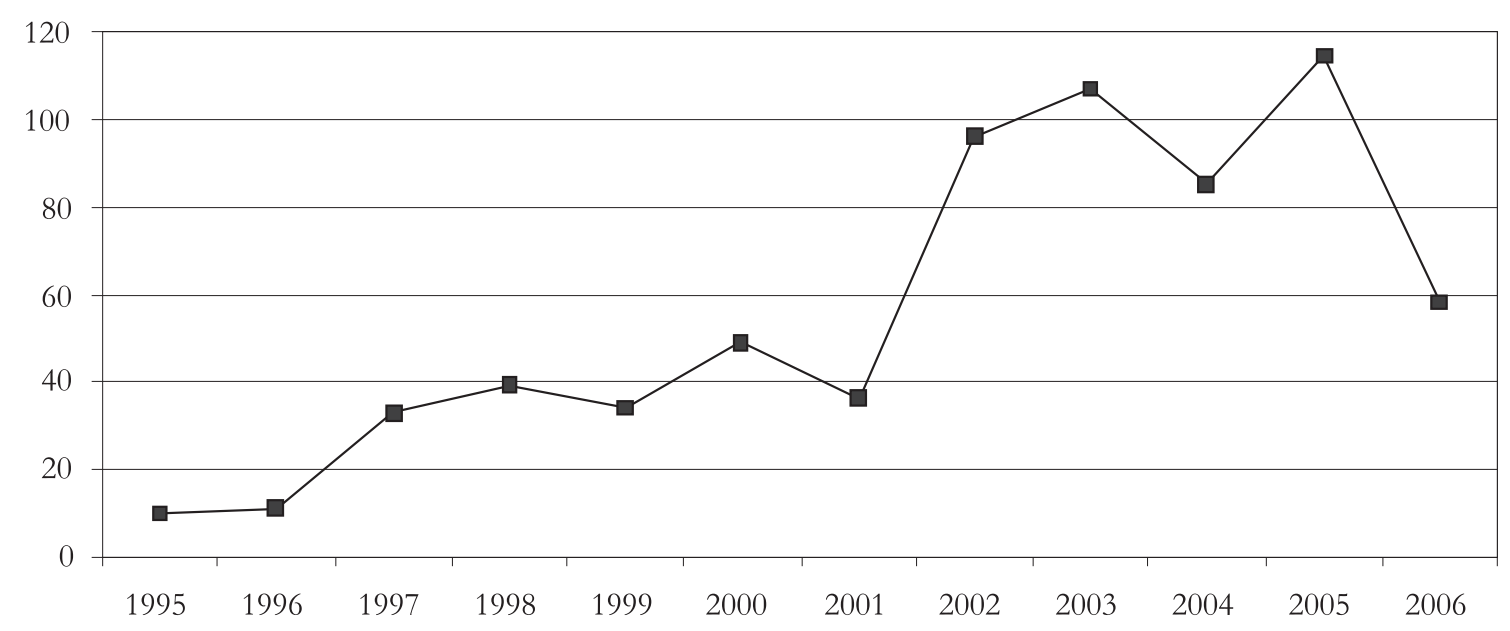

uma demanda represada de tecnologias passíveis de patenteamento, uma vez que até então toda a ênfase acadêmica baseava-se nas publicações.

A queda ocorrida nos anos de 2004 e 2006 mostra a oscilação dos depósitos universitários. A queda expressiva de depósitos ocorrida em 2006 está relacionada também à incompletude da base para aquele ano. Há um atraso entre os depósitos efetuados no Inpi e a disponibilização de seus registros na base, principalmente as patentes depositadas sob o Tratado de Cooperação em Matéria de Patentes (TCP).

A figura 2 reflete a participação de cada universidade no total de depósitos de patentes efetuados entre os anos de 1995-2006 pelas universidades abarcadas por esta pesquisa. A Unicamp é a responsável pela maior parcela dos depósitos realizados entre as universidades públicas paulistas, com $60 \%$ dos registros. Isso se deve à atenção que essa universidade tem dado historicamente à questão e, mais recentemente, à atuação da agência Inova Unicamp, criada em 2003, que tem desempenhado importante papel na implementação de uma cultura voltada para a proteção patentária (CASTRO, JANNUZZI; MATTOS, 2007; FAPESP, 2007).

A segunda instituição com maior número de depósitos é a Universidade de São Paulo. A USP tem participação de $24 \%$ dos depósitos realizados no período estudado. Levando em consideração a estrutura da USP, sua produção científica, números de pesquisadores atuantes e tempo de existência, os depósitos de patentes são bem 
menos expressivos se comparados com os números da Unicamp. Conforme apresentado na tabela 1 , o número de pesquisadores atuantes na Universidade de São Paulo nos últimos anos sempre esteve acima do dobro de pesquisadores da Universidade Estadual de Campinas.

A Unesp é responsável por 5\% dos depósitos realizados, ocupando a terceira posição entre as universidades analisadas. UFSCar e Unifesp são as universidades com menor número de depósitos realizados, responsáveis, respectivamente, por $5 \%$ e $4 \%$ do total dos registros recuperados no período.

FIGURA 3

Natureza dos depósitos conjuntos das universidades públicas paulistas (1995-2006)

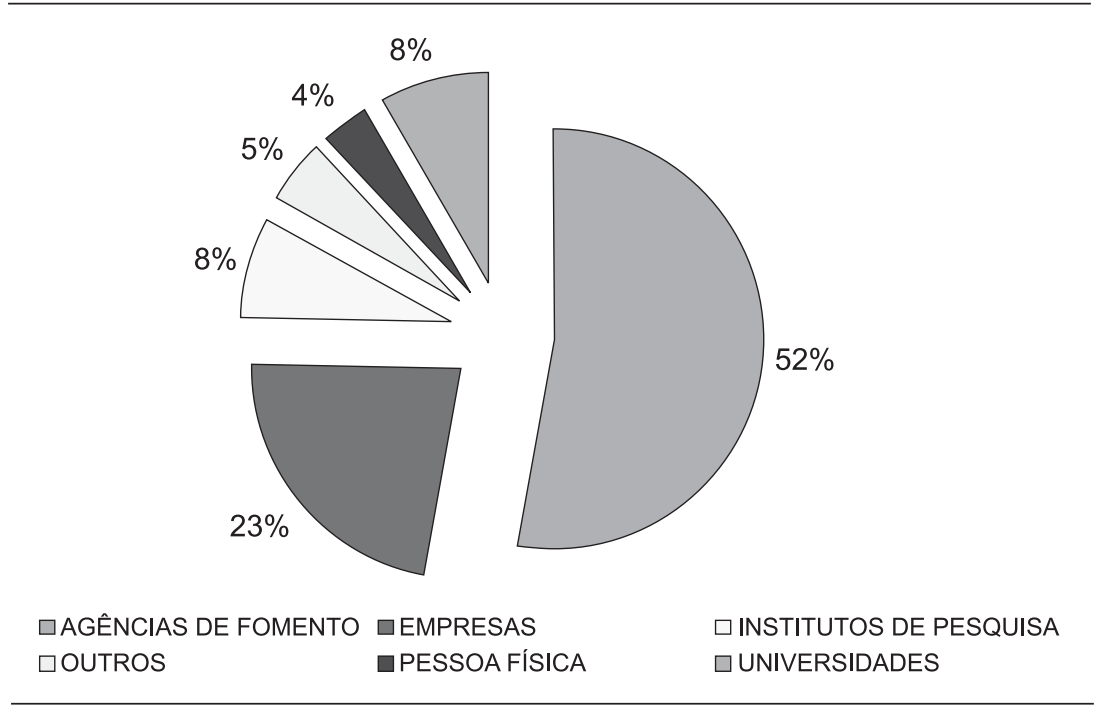

As principais instituições que cooperam com as universidades estudadas são de natureza pública. Apenas 19\% dos depósitos realizados pelas universidades públicas paulistas foram realizados em conjunto com outras instituições. Desse total, 52\% com agência de fomento. No caso das universidades estudadas, a Fapesp foi a única agência com a qual ocorreram depósitos dessa natureza. As empresas foram responsáveis por $23 \%$ dos depósitos realizados em parceria. Considerando o total de depósitos, com e sem parceria, a porcentagem deles com empresas não chega a 5\% do total. Demais universidades e institutos de pesquisa participam juntos de 16\% dos depósitos com as universidades paulistas, com 8\% para cada instituição. Pessoas físicas e outros tipos de parceiros somam juntos $9 \%$ de participação (figura 3).

É intuitivo pensar que a quantidade de depósitos gerados pelas universidades possa estar relacionada diretamente ao número de pesquisadores que ela possui; no entanto a tabela 1 , a seguir, apresenta uma situação divergente a respeito da produtividade dos pesquisadores em termos de pedidos de patentes. No ranking das universidades que mais depositaram, a Unicamp é expressivamente a principal responsável pelos depósitos das universidades estudadas, porém possui menos pesquisadores que USP e Unesp.

Ao calcularmos a proporção entre os pesquisadores atuantes nas universidades e o número de depósitos de patentes gerados durante um período de sete anos, chega-se a resultado interessante. $\mathrm{Na}$ relação pesquisador/depósito de patente, a Unesp se apresentou como a instituição com menor produtividade, necessitando de 63,3 pesquisadores para que um depósito de patente fosse realizado durante o período de um ano. A USP, segunda instituição que mais depositou patentes no período abrangido por esta pesquisa, foi a segunda universidade com menor produtividade na reação pesquisador/depósito de patente, tendo um depósito para cada 51,81 pesquisadores. As duas universidades federais, UFSCar e Unifesp, que realizaram menos depósitos de patentes em números absolutos, demonstraram ter maior produtividade, com um depósito efetuado para cada 31,78 e 29,07 pesquisadores, respectivamente.

Já a Unicamp apresenta números surpreendentes, gerando um depósito de patente a cada 7,97 pesquisadores, destacando-se como a universidade 
TABELA 1

Relação depósito/pesquisador

\begin{tabular}{|c|c|c|c|c|c|c|c|}
\hline & \multicolumn{4}{|c|}{ Número de pesquisadores } & \multirow{2}{*}{$\begin{array}{c}\text { Média } \\
\text { pesquisadores } \\
2000-2006\end{array}$} & \multirow{2}{*}{$\begin{array}{l}\text { Depósitos } \\
\text { de patentes } \\
\text { acumulados } \\
2000-2006\end{array}$} & \multirow{2}{*}{$\begin{array}{c}\text { Número de } \\
\text { pesquisadores / } \\
\text { depósitos de } \\
\text { patentes 2000- } \\
2006\end{array}$} \\
\hline & 2000 & 2002 & 2004 & 2006 & & & \\
\hline USP & 5.173 & 5.085 & 7.794 & 8.478 & $6.632,5$ & 128 & 51,81 \\
\hline Unesp & 1.680 & 2.440 & 3.346 & 3.944 & $2.852,5$ & 45 & 63,3 \\
\hline Unicamp & 2.015 & 2.263 & 2.903 & 3.253 & $2.608,5$ & 327 & 7,97 \\
\hline UFSCar & 647 & 723 & 928 & 1.135 & 858,25 & 27 & 31,78 \\
\hline Unifesp & 582 & 628 & 866 & 932 & 610,5 & 21 & 29,07 \\
\hline
\end{tabular}

Fonte de número de pesquisadores: Adaptado de CNPq - Conselho Nacional de Desenvolvimeto Científico e Tecnológico. Súmula estatística do Diretório dos Grupos de Pesquisa no Brasil. Brasília, DF: CNPq, 2007.

com melhor desempenho na relação pesquisador/ depósito de patente. Vale ressaltar que essa relação de produtividade pode ser consequência, além das áreas de atuação de cada universidade, de diferenças existentes entre as políticas internas de cada universidade, já que a seleção das tecnologias patenteáveis pode ser mais rígida em uma instituição que em outra, refletindo assim no total de depósitos gerados (GARNICA, 2007).

A tabela 2 apresenta a relação do número de depósitos de patentes com a quantidade de programas de pósgraduação existente em cada universidade.

A USP, entre os anos de 2000 e 2006, contabilizou, em média, 217 programas de pós-graduação por ano. No mesmo período, foram registrados 128 depósitos de patentes, perfazendo 0,59 depósito por programa. Com 21 depósitos realizados no período e média de 40,86 programas de pós-graduação, a Unifesp apresentou média de 0,51 depósitos de patente por programa. Para a Unesp, a relação de depósito de patente/programa de pós-graduação é de 0,46. Unicamp e UFSCar são as únicas instituições que possuem número de depósitos maior que a quantidade de programas de pós-graduação entre os anos de 2000 e 2006. Nesse período, a Unicamp teve média de 5,16 depósitos por programa, enquanto a UFSCar obteve 1,45 (tabela 2).

Embora este trabalho não tenha levantado especificamente as patentes geradas por programa de pós-graduação e a despeito do fato de tais programas pertencerem a diferentes áreas nas

TABELA 2

Relação depósito/programa de pós-graduação

\begin{tabular}{|c|c|c|c|c|c|c|c|c|c|c|}
\hline & \multicolumn{7}{|c|}{ Número de programas de pós-graduação } & \multirow{2}{*}{$\begin{array}{c}\text { Média } \\
\text { programa } \\
\text { pós- } \\
\text { graduação }\end{array}$} & \multirow{2}{*}{$\begin{array}{l}\text { Depósitos de } \\
\text { patentes } \\
\text { acumulados } \\
2000-2006\end{array}$} & \multirow{2}{*}{$\begin{array}{c}\text { Depósito } \\
\text { por } \\
\text { programa }\end{array}$} \\
\hline & 2000 & 2001 & 2002 & 2003 & 2004 & 2005 & 2006 & & & \\
\hline USP & 214 & 216 & 217 & 220 & 215 & 220 & 223 & 217,86 & 128 & 0,59 \\
\hline Unesp & 92 & 94 & 95 & 97 & 98 & 100 & 104 & 97,14 & 45 & 0,46 \\
\hline Unicamp & 61 & 62 & 64 & 62 & 65 & 65 & 65 & 63,43 & 327 & 5,16 \\
\hline UFSCar & 17 & 17 & 18 & 18 & 19 & 20 & 21 & 18,57 & 27 & 1,45 \\
\hline Unifesp & 38 & 35 & 36 & 42 & 45 & 45 & 45 & 40,86 & 21 & 0,51 \\
\hline
\end{tabular}

Fonte do número de programas de pós-graduação: Coordenação de Aperfeiçoamento de Pessoal de Nível Superior (Capes). Estatísticas da pós-graduação. Brasília, DF: Capes, 2006c. Disponível em: http://www.capes.gov.br/sobre/estatisticas>. Acesso em: 21 ago. 2008. 
diversas universidades estudadas, a relação entre esses dados se mostra pertinente, já que a pós-graduação nas universidades representa a espinha dorsal da pesquisa científica, capaz de gerar conhecimento novo a ser absorvido pela sociedade.

Ao fazermos um ranking das universidades por produtividade (depósito de patente) de seus programas de pós-graduação, temos Unicamp $(5,16)$, UFScar $(1,45), \operatorname{USP}(0,59)$, Unifesp $(0,51)$ e Unesp $(0,46)$ (tabela 2).

Outro aspecto de análise interessante é o confronto do número de publicação científica das universidades com o número de depósitos de patentes. A tabela 3 mostra o total de publicações das universidades indexadas na base Science Citation Index Expanded (SCIE) entre os anos de 1998 a 2002 (FAPESP, 2005). Na segunda coluna da tabela, é apresentada a quantidade de depósitos de patentes realizados pelas universidades no mesmo período.

Os valores apresentados na última coluna da tabela 3 correspondem ao número de artigos gerados para cada depósito de patente. No caso da Unifesp, entre os anos de 1998 a 2002 foi gerado um depósito de patente a cada 422,67 artigos publicados. Já a Unicamp teve um depósito de patente para cada 40,35 artigos publicados.

Não foi objetivo deste trabalho correlacionar as publicações geradas pelas universidades às patentes por elas depositadas. A relação apresentada na tabela 3 apenas permite a observação da proporção entre a produção científica e a produção tecnológica das universidades. Os números evidenciam a primazia das publicações científicas, mesmo no caso da Unicamp, em que o volume de pedidos de patentes é consideravelmente maior.

A figura 4, a seguir, revela as atividades econômicas, através dos domínios tecnológicos (classificação da OST), dos depósitos de patentes gerados nas universidades. Entre as cinco áreas de classificação, os registros estão concentrados nas áreas de "química fina e farmácia", "procedimento químico de base metalúrgica" e "instrumentação". Essa característica revela que os depósitos feitos pelas universidades têm como suporte as ciências básicas, principalmente relacionadas com química e física. Química também se destaca nos indicadores Fapesp do ano de 2004, aparecendo como a terceira área do conhecimento com maior número de publicações científicas das instituições paulistas nos anos de 1998-2002, indexadas na base Web of Science. A primeira e segunda posição pertence, respectivamente, às áreas de medicina e física. As publicações brasileiras indexadas na base SCIE estiveram concentradas nas áreas de medicina, física e química, cada uma com contribuição de $25,3 \%$, $14,1 \%$ e $10,4 \%$, respectivamente (FAPESP, 2005).

Importante salientar que as universidades possuem patentes depositadas em todos os domínios tecnológicos, tendo destaque as áreas de "química fina e farmácia", "procedimento químico de base metalúrgica" e "instrumentação".

TABELA 3

Relação publicação versus depósito de patente

\begin{tabular}{l|c|c|c}
\hline & $\begin{array}{c}\text { Publicações* } \\
(\mathbf{1 9 9 8 - 2 0 0 2 )}\end{array}$ & $\begin{array}{c}\text { Depósitos de } \\
\text { Patentes (1998-2002) }\end{array}$ & $\begin{array}{c}\text { Relação } \\
\text { Publicação/Depósito } \\
\text { de Patente }\end{array}$ \\
\hline USP & 1.6517 & 51 & 323,86 \\
\hline Unicamp & 6778 & 168 & 40,35 \\
\hline Unesp & 4335 & 20 & 216,75 \\
\hline Unifesp & 2536 & 6 & 422,67 \\
\hline UFSCar & 1869 & 9 & 207,67 \\
\hline
\end{tabular}

*Publicações indexadas na base SCIE - 1998-2002 (FAPESP, 2005) 


\section{CONCLUSÕES}

Este trabalho teve o objetivo de realizar análise exploratória dos depósitos de patentes realizados pelas universidades públicas paulistas. Verificamos o adiantamento da Unicamp em relação às demais universidades na quantidade de patentes depositadas, responsável por $60 \%$ do total de depósitos. Outra característica bastante interessante encontrada é a diversidade das áreas em que os depósitos estão sendo realizados. As áreas das ciências básicas, como química e física, mostraram ser o suporte da pesquisa acadêmica na geração de tecnologias patenteáveis. A Unicamp, além de ter se apresentado como a principal depositante em números absolutos, possui maior número de patentes por pesquisador e por programa de pós-graduação, bem como mais depósitos gerados por publicação científica.

Paralelamente ao desempenho indiscutível alcançado pela Unicamp, outros números significativos foram identificados. As duas universidades federais, UFSCar e Unifesp, apresentaram desempenho superior à USP e Unesp na quantidade de depósito por pesquisador. AUFSCar também possui o segundo melhor desempenho na relação dos depósitos com a quantidade de programa de pós-graduação.

Vale ressaltar que a heterogeneidade das instituições estudadas é fator que deve ser considerado na análise dos resultados. Instituições como USP e Unicamp têm seus programas de pós-graduação concentrados nas áreas de ciências da saúde, ciências exatas e engenharias. A pós-graduação da Unifesp é predominantemente na área de ciências da saúde. Já UFSCar e Unesp apresentam uma divisão equilibrada entre seus programas de pós-graduação, tendo destaque áreas como ciências humanas. Estas características certamente influenciam no número de depósitos realizados pelas universidades, já que algumas áreas estão mais voltadas para geração de tecnologias que outras.

Não é expressiva a quantidade de depósitos realizados em parceria. A universidade se apresenta como única titular das patentes em mais de $80 \%$ dos casos. E, quando identificados quais são esses parceiros, a Fundação de Amparo à Pesquisa do Estado de São Paulo, maior fonte de financiamento de pesquisa no Estado, aparece como principal cotitular nas tecnologias que geram patentes.

O fato é que se verifica um avanço, mesmo instável, no número de patentes advindas das universidades, embora se tenha muito a evoluir neste aspecto. As universidades possuem enorme potencial na geração de produtos e processos que podem ser empregados no setor industrial, ajudando no preenchimento da lacuna existente no desenvolvimento de tecnologias aplicáveis ao mercado por parte das empresas, fazendo por assim cumprir a recém-criada Lei de Inovação e consolidar o processo de inovação no país.

As universidades também têm demonstrado maior preocupação na gestão da propriedade intelectual em suas atividades. A criação de agências internas de inovação permitiu a conscientização e apoio a 
pesquisadores para que encaminhem suas pesquisas pensando na patente como um dos subprodutos possíveis.

Ante a considerável contribuição dos indicadores patentários na construção de uma política nacional de inovação, cabe ressaltar a necessidade de se ter uma base nacional de patentes de mais fácil utilização e com possibilidade de consulta de indicadores mais atualizados e integrados com outros já existentes, contribuindo na divulgação e mapeamento das tecnologias disponíveis. O cenário atual exige constante monitoramento da geração tecnológica do país e esforços ilimitados na construção de um canal direto de transferência dessa tecnologia ao setor produtivo, resguardando os direitos das universidades e dos inventores.

Diante dos resultados alcançados nesta pesquisa e de suas limitações, cabe apresentar algumas sugestões para novos estudos que possam completar e aprofundar o entendimento do tema abordado neste trabalho. Sugere-se a ampliação do universo estudado, abrangendo as demais universidades públicas existentes e possíveis comparações com universidades privadas que também possuam depósitos de patentes. Analisar a política interna das instituições a fim de confrontar os resultados empíricos obtidos nesta pesquisa e levantar os principais fatores que fazem com que as universidades possuam diferentes desempenhos no tratamento da propriedade da propriedade intelectual também enriqueceria o entendimento deste tema.

Artigo submetido em 30/10/2008 e aceito em 18/08/2009.

\section{REFERÊNCIAS}

AGÊNCIA BRASILEIRA DE DESENVOLVIMENTO INDUSTRIAL (ABDI). Projeto metodologia pra conceber e executar. Plano de Mobilização Brasileira pela Inovação - MOBIT. Brasília, DF: ABDI, 2007. 51 p. Relatório final do projeto. Disponível em: $<$ http://www.abdi.com.br/?q=system/files/Relatorio_Final.pdf $>$. Acesso em: 23 jul. 2008.

CASTRO, A. C.; JANNUZZI, C. A. S. C.; MATTOS, F. A. M. de. Produção e disseminação de informação tecnológica: a atuação da Inova - Agência de Inovação da UNICAMP. Transinformação, Campinas, v. 19, n. 3, p. 265-277, set./dez. 2007.
ETZKOWITZ, H. Research groups as "quase-firms": the invention of the entrepreneurial university. Research Policy, Amsterdam, v. 32, n. 1, p. 109-121, Jan. 2003.

FUNDAÇÃO DE AMPARO À PESQUISA DO ESTADO DE SÃO PAULO (FAPESP). Agência FAPESP. Primeira em patentes. São Paulo: FAPESP, 2007. Disponível em: <http://www.agencia.fapesp. $\mathrm{br} / \mathrm{materia} / 8217 /$ especiais/primeira-em-patentes.htm>. Acesso em: 3 jun. 2009.

- Indicadores de ciência, tecnologia e inovação em São Paulo 2004. São Paulo: [s.n.], 2005, 992 p.

GARNICA, L. A. G. Transferência de tecnologia e gestão da propriedade intelectual em universidades públicas no estado de São Paulo. 2007. 203 f. Dissertação (Mestrado em Engenharia de Produção)-Universidade Federal de São Carlos, São Carlos, 2007.

HAASE, H.; ARAÚJO, E. C. de; DIAS, J. Inovações vistas pelas patentes: exigências frente às novas funções das universidades. Revista Brasileira de Inovação, Rio de Janeiro, v. 4, n. 2, jul./dez. 2005.

HSU, C. W. Formation of industrial innovation mechanisms through the research institute. Technovation, Oxford, v. 25, n. 11, p. 1317-1329, Nov. 2005.

INSTITUTO NACIONAL DE PROPRIEDADE INDUSTRIAL (INPI). Universidades brasileiras: utilização do sistema de patentes de 2002 a 2004. Rio de Janeiro, 2007. 46 p. Disponível em: <http:// www.inpi.gov.br/menu-esquerdo/informacao/pdf-dos-estudos/ Universidades $\% 20$ Brasileiras $\% 20$ - $\% 20$ Utilizacao $\% 20$ do $\% 20$ Sistema $\% 20$ de $\% 20$ Patentes $\% 2$ 0de $\% 202000 \% 20 \mathrm{a} \% 202004$.pdf>. Acesso em: 2 ago. 2008.

LOPES, N. P\&D, propriedade intelectual e comercialização da tecnologia. In: ENCONTRO DA PROPRIEDADE INTELECTUAL E COMERCIALIZAÇÃO DA TECNOLOGIA, 2. 1999. Rio de Janeiro. [Anais]... Rio de Janeiro: Rede de Tecnologia do Rio de Janeiro, 1999. $100 \mathrm{p}$.

MOWERY, D. C.; ROSENBERB, N. Trajetórias da inovação: a mudança tecnológica nos Estados Unidos da América no século XX. Campinas: Editora da UNICAMP, 2005. 230 p.

ORGANIZATION FOR ECONOMIC COOPERATION AND DEVELOPMENT (OECD). Manual de Oslo: diretrizes para coleta e interpretação de dados sobre inovação. 3. ed. Tradução da Financiadora de Estudos e Projetos. Rio de Janeiro: FINEP, 1997. $184 \mathrm{p}$.

SABINO, L. S. Caracterização da proteção às patentes como estímulo ao desenvolvimento econômico. 2007. Dissertação (Mestrado em Direito)Universidade Católica de Brasília, Brasília, 2007.

TORKOMIAN, A. L. V. Gestão de tecnologia na pesquisa acadêmica: o caso de São Carlos. 1997. 303 f. Tese (Doutorado em Administração)Universidade de São Paulo, São Paulo, 1997.

TORKOMIAN, A. L. V. Panorama dos núcleos de inovação tecnológica no Brasil. In: SANTOS, M. E. R.; TOLEDO, P. T. M.; LOTUFO, R. A. (Org.). Transferência de tecnologia. Campinas: Komedi, 2009. p. 21-37.

WORLD INTELLECTUAL PROPERTY ORGANIZATION (WIPO). Geneva, 2007. Disponível em: <http://www.wipo.org>. Acesso em: 10 abr. 2007.

Ci. Inf., Brasília, v. 38, n. 2, p. 9-18, maio/ago. 2009 